\title{
Battery-State Dependent Power Control as a Dynamic Game
}

\author{
Ishai Menache* and Eitan Altman ${ }^{\dagger}$ \\ * Faculty of Electrical Engineering, Technion, Haifa 32000, Israel \\ $\dagger$ INRIA, Sophia-Antipolis, 2004 Route des Lucioles, 06902 Sophia-Antipolis Cedex, France
}

\begin{abstract}
Consider an uplink cellular network shared by a finite number of mobile users with limited batteries. Whenever the battery drains out, the user pays a fixed price to recharge the battery. Users, assumed to have always traffic to send, control their transmission power in a noncooperative way. The novelty of our model is in considering the dynamic game in which the transmission power of a player may depend on the amount of energy left in its battery. We consider various models and various types of constraints and derive for each one the structure of the equilibrium. A particular interesting structure is obtained when there are constraints on the maximum transmission power which become tighter as the battery drains out. Using Schur convexity and majorization, we identify an equilibrium where each mobile distributes the power of each battery along the battery's lifetime in a way equivalent to a max-min assignment.
\end{abstract}

\section{Index Terms}

Dynamic power control; Nash equilibrium; Battery lifetime; Schur convexity and Majorization, Max-min fairness.

\section{INTRODUCTION}

We examine how mobiles, aware of their remaining energy, adjust their individual power discipline. The performance of each mobile in multi-user wireless networks is significantly affected by the actions taken by other mobiles. It is well known, for example, that a station that transmits at high power prevents the signal of other stations from being identified at the base station. In recent years it has been advocated that for scalability reasons, mobiles should have the freedom to distributively adjust their transmission parameters (such as the transmission power) for optimizing their own performance. Much research has therefore been devoted over the last decade for the study of wireless networks through game-theoretic tools. In particular, several papers (e.g., [1]-[4]) have studied the equilibrium properties of power allocation games. The above papers consider a static setup, where mobiles adjust their power based on average network conditions. Consequently, an equilibrium is characterized by fixed transmission powers of all users. Recently, [5], [6] consider the power allocation game under time-varying channel qualities, which require users to adjust their transmission power as a function of the channel state. These games result in stationary transmission strategies that are essentially a mapping between the possible channel states and the set of power levels. In some of the above references, there is an upper bound constraint on the average power investment of the users. To the best of our knowledge, however, the power-control game literature lacks the explicit incorporation of the dynamic affect of transmissions on the lifetime of the battery.

In this work we initiate the study of the consequences of the dynamic change in battery-energy on the transmission strategies and throughput of wireless nodes. Our network model consists of a finite number of mobile users who transmit to a common base station. Every time that the energy of the battery drains out, the user pays a fixed price for recharging the battery. Users, assumed to have always traffic to send, control their transmission power in order to maximize their individual net payoff, which consists of the average throughput (or its utility) minus the recharge costs. We study here the existence, uniqueness and structure of the equilibrium. To that end, we consider a variety of possible network configurations, including continuous or slotted time models, continuous or discrete power levels, and more.

Contribution and Organization. The main results of this work are summarized below.

- We introduce a novel distributed power control problem, where users base their transmission decisions on the amount of energy remaining in their battery. 
- We prove the existence of an equilibrium point for several network models. In particular, there always exists a special equilibrium point where users employ regular transmission strategies. Roughly, regular strategies are such that the variance of the transmission-power sequence is kept minimal. In some cases, the regular strategy equilibrium reduces to fixed transmission powers for all mobiles.

- We introduce and study a novel framework for power control in cases that battery-dependent constraints are imposed on the transmission power.

- We show that the equilibrium is in general not unique. This is contrast to most power control games we are aware of in the literature, where uniqueness holds (e.g., [1], [2], [5]).

Besides the analytical results, we introduce some novel tools for the analysis of power-control dynamic games. In particular, we apply the theory of majorization and Schur convexity (see Section VI) for deriving the mobile optimal transmission strategies and proving the existence of an equilibrium.

The organization of the paper is as follows. We present the network model in Section II, addressing both continuous and discrete time settings. In Section III we show some basic properties that are common to both time models. Sections IV and V focus on the existence of an equilibrium point under continuous time and discrete time, respectively. In Section VI we consider the case where additional constraints are imposed on the power selection. Section VII discusses other user utilities in the context of our model. In Section VIII we show that other equilibria, besides the regular one, may exist. Conclusions and further research directions are drawn in Section IX.

\section{THE MODEL}

Basic Definitions. We consider a finite set of $N$ terminals $j=\{1, \ldots, N\}$ controlling their transmission power. Each terminal $j$ has an amount of energy $\mathcal{E}^{j}$ when its battery is new (e.g., at time 0 ). The terminal is assumed to have always packets to transmit. Each terminal implements a power control policy where the transmission power is allowed to depend on the energy level of its battery. We shall consider a dynamic power control problem assuming that the time between two successive replacements of batteries by terminal $j$ is at least some constant $s^{j}$ and at most a constant $S^{j}$. We assume that a battery is replaced only when it is completely empty. When terminal $j$ replaces a battery then it receives a new battery with the same energy of $\mathcal{E}^{j}$ for a cost of $C^{j}$ units. A terminal thus controls both the power as well as the time at which the batteries are changed.

The basic quantity that determines the instantaneous throughput of each terminal is the signal to interference plus noise ratio (SINR) at the base station. Let $P_{t}^{j}$ denote the transmission power of terminal $j$ at time $t$. The SINR at time $t$ is given by

$$
\operatorname{SINR}_{t}^{j}=\frac{L^{j} P_{t}^{j}}{N_{0}+\sum_{i \neq j} L^{i} P_{t}^{i}}
$$

where $L^{j}$ is the gain between transmitter $j$ and the common base station, and $N_{0}$ is the ambient noise power. We shall assume that $L^{j}$ are constant in time. We shall further assume that player $j$ does not observe the battery state of other users nor their transmission powers.

For each terminal $j$, let $h^{j}(\gamma)$ be a continuous, concave increasing function representing the benefit for transmitting with an SINR of $\gamma$. For example, the special case of $h^{j}(\gamma)=\log (1+\gamma)$ corresponds to the throughput obtained under a Gaussian channel, assuming capacity achieving codes. We shall assume in this paper that each terminal wishes to maximize a time average value of $h^{j}(\gamma)$ minus the recharge costs of the battery. Using game-theoretic terminology, we shall refer to this measure as the terminal (or user) utility. In order to formalize the user utility we require the following notations.

- $\zeta_{t}^{j}$ - the number of times that the $j$ th terminal has changed battery by time $t$.

- $\eta_{m}^{j}$ - the time when the battery of terminal $j$ is changed for the $m$ th time.

- $\tau_{m}^{j}$ - the total lifetime of the $m$ th battery of terminal $j$.

We shall consider in this paper both continuous and discrete time models, as outlined below.

Continuous Time Model. Mobiles are allowed to adjust their power at any time $t$. The terminal selection of powers over time would be often referred to as the terminal's strategy (or policy). We denote by $u^{j}$ the strategy of mobile $j$, and by $u=\left(u^{1}, \ldots, u^{N}\right)$ the vector of strategies. Then the additive utility described above is given by

$$
J^{j}(u)=\underline{\lim }_{T \rightarrow \infty} \frac{1}{T} \int_{t=0}^{T} h^{j}\left(\operatorname{SINR}_{t}^{j}\right) \mathrm{d} t-C^{j} \zeta_{T}^{j} .
$$


We may reformulate an equivalent utility, embedded at times in which the battery is changed.

Let $H_{m}^{j}=\int_{\eta_{m}^{j}}^{\eta_{m+1}^{j}} h^{j}\left(\mathrm{SINR}_{t}^{j}\right) d t$. Due to the finite bound on the battery lifetime, the utility in (2) is equivalent to

$$
\tilde{J}^{j}(u)=\underline{\lim }_{n \rightarrow \infty} \frac{\sum_{m=1}^{n}\left(H_{m}^{j}-C^{j}\right)}{\sum_{m=1}^{n} \tau_{m}^{j}} .
$$

Discrete Time Model. Time is divided to integer slots: $t=\{1,2, \ldots\}$. At time $t$ each terminal $j$ chooses a power of $P_{t}^{j}$ for transmission, which is kept constant until the next slot. As a result, the remaining energy in the battery decreases by $P_{t}^{j}$. Note that a feasible strategy $u^{j}$ should use power levels that do not exceed the current battery energy. The corresponding utility for player $j$ is

$$
J^{j}(u)=\underline{\lim }_{T \rightarrow \infty} \frac{1}{T} \sum_{t=1}^{T} h^{j}\left(\operatorname{SINR}_{t}^{j}\right)-C^{j} \zeta_{T}^{j} .
$$

Equation (3) holds in the discrete setting as well, however with $H_{m}^{j}=\sum_{\eta_{m}^{j}}^{\eta_{m}^{j}-1} h^{j}\left(\operatorname{SINR}_{t}^{j}\right)$.

Nash Equilibrium. As the SINR of each mobile depends on the power decisions of other mobiles, we formulate the distributed decision problem (under both continuous and discrete time models) as a non-cooperative game between the $N$ mobiles.

Definition 2.1: We say that $u$ is a Nash equilibrium [7] if

$$
J^{j}(u) \geq J^{j}\left(\tilde{u}^{j}, u^{-j}\right), \quad j \in\{1, \ldots, N\}
$$

for any strategy $\tilde{u}^{j}$, where $u^{-j}$ stands for the vector of strategies of all users but the $j$ th one.

User Actions. For the analysis of the noncooperative game defined above, it is sometimes convenient to construct a decision model for each terminal $j$ embedded at the moments when its battery is changed. An action $\alpha^{j} \in \mathcal{A}^{j}$ for terminal $j$ is defined as a vector $\alpha^{j}=\left(\nu(j), P_{t}^{j}, \forall 0<t \leq \nu(j)\right)$, where $\nu(j)$ denotes the time till the battery is changed next (taking values in the interval $\left[s^{j}, S^{j}\right]$ ), and $P_{t}^{j}$ is the power used by terminal $j$ when the battery has age $t$. We use the same notation $\alpha^{j}$ for both time models, yet recall that the transmission power in discrete time cannot exceed the total energy remaining in the battery. We denote by $\mathcal{A}^{j}(s)$ the set of actions among $\mathcal{A}^{j}$ for which $\nu(j)=s$.

\section{Basic Structural Results for Both Time Models}

We focus in this section on several special cases for which the existence of a Nash equilibrium (5) can be obtained quite immediately, for both continuous and discrete time. To that end, we introduce the following assumptions:

- Assumption Q1(j): The periods for changing the batteries for terminal $j$ are fixed. In other words, $s^{j}=S^{j}$.

- Assumption Q2(j): All terminals but $j$ use constant powers, i.e., for all $i \neq j$ there are constants $P^{i}$ such that $P_{t}^{i}=P^{i}$ for all $t$.

Lemma 3.1: Assume Q2(j) for some $j$. Then terminal $j$ has a best response strategy (i.e., one that maximizes $J^{j}$ ) that uses a constant power at any time.

Proof: For any fixed sequence $\eta_{m}^{j}$, the utility for terminal $j$ is maximized by maximizing $H_{m}^{j}$ (in the enumerator of the utility function corresponding to the embedded problem (3)) for each $m$. Let $\Theta$ be uniformly distributed on $\left[\eta_{m}^{j}, \eta_{m+1}^{j}\right)$. Denote by $E_{\theta}$ the expectation with respect to this distribution, and let $e_{m}^{j}$ denote the total energy that terminal $j$ uses in period $m$. Note that $E_{\theta}\left[P_{\Theta}^{j}\right]=\frac{e_{m}^{j}}{\tau_{m}^{j}}$. Now, $H_{m}^{j}$ can be written as $H_{m}^{j}=\tau_{m}^{j} E_{\theta}\left[h^{j}\left(\frac{L^{j} P_{\Theta}^{j}}{N_{0}+\sum_{i \neq j} L^{i} P^{i}}\right)\right]$. Applying Jensen's inequality, we get: $H_{m}^{j} \leq \tau_{m}^{j} h^{j}\left(E_{\theta}\left[\frac{L^{j} P_{\Theta}^{j}}{N_{0}+\sum_{i \neq j} L^{i} P^{i}}\right]\right)=\tau_{m}^{j} h^{j}\left(\frac{L^{j} e_{m}^{j} / \tau_{m}^{j}}{N_{0}+\sum_{i \neq j} L^{i} P^{i}}\right)$. We conclude that $H_{m}^{j}$ is maximized by choosing equal transmission powers during the lifetime of the $m$ th battery. They should be chosen so as to sum to $\mathcal{E}^{j}$. It remains to determine $\tau_{m}^{j}$. The utility (3) is clearly maximized by choosing $\tau_{m}^{j} \in \mathcal{R}$ for all $m$ where $\mathcal{R}:=\operatorname{argmax}_{r \in\left[s^{j}, S^{j}\right]} \frac{H^{j}\left(\mathrm{SINR}^{j, r}\right)-C^{j}}{r}$, and where $H^{j}\left(\mathrm{SINR}^{j, r}\right)=r h^{j}\left(\frac{L^{j} \mathcal{E}^{j} / r}{N_{0}+\sum_{i \neq j} L^{i} P^{i}}\right)$. Since the set of possible lifetimes is compact (in both continuous and discrete time), there is indeed a best response that uses a constant power of $\mathcal{E}^{j} / R$ for some $R \in \mathcal{R}$.

The next corollaries follow directly from the above lemma.

Corollary 3.2: Assume that Q1(j) holds for all $j$. Then there exists an equilibrium in which for each terminal $j, P_{t}^{j}$ do not depend on $t$ and are given by $\mathcal{E}^{j} / R^{j}$. 
Proof: Immediate from Lemma 3.1 and (5).

Corollary 3.3: Assume that $C^{j}=0$ for all $j$. Then there exists an equilibrium in which for each terminal $j, P_{t}^{j}$ do not depend on $t$ and are given by $\mathcal{E}^{j} / s^{j}$.

Proof: Assume that all players other than $j$ use transmission powers that are constant in $t$. Consider the embedded model with additive utility. Then Lemma 3.1 implies that the optimal response policy for player $j$ has the form $\alpha_{n}^{j}=\left[R^{j}, \mathcal{E}^{j} / R^{j}, \ldots, \mathcal{E}^{j} / R^{j}\right]$. Hence the best response for player $j$ is a fixed power. Among all strategies $u^{j}$ for which the power is constant in time, the utility of player $j$ is obviously maximized with $R^{j}=s_{j}$, since the SINR is maximal with no battery costs involved. Hence at equilibrium we have $R^{j}=s^{j}$ for every $j$, and the transmission power is constant, given by $\mathcal{E}^{j} / R^{j}$.

\section{Equilibrium For the Continuous-Time Model}

In this section we prove the existence of an equilibrium point (5) for the continuous time model. Specifically, we show that there always exists an equilibrium point where all terminals use constant transmission powers. We then show that this particular equilibrium is unique for several utilities that are often used in the literature. The main result of this section is summarized below.

Theorem 4.1: There exists an equilibrium point for the continuous time model. Specifically, there exists an equilibrium in which $P_{t}^{j}$ do not depend on $t$ for every terminal $j$, and are given by $\mathcal{E}^{j} / R^{j}$, where $R^{j}$ is some constant satisfying $s^{j} \leq R^{j} \leq S^{j}$.

Proof: Assume that each terminal $j$ uses a constant period $R^{j}$ and a constant power of $\mathcal{E}^{j} / R^{j}$. In other words, $\alpha^{j}=\left[R^{j}, \mathcal{E}^{j} / R^{j}, \ldots, \mathcal{E}^{j} / R^{j}\right]$. Then the utility of terminal $j$ is given by

$$
J^{j}\left(R^{j}\right)=h^{j}\left(\frac{L^{j} \mathcal{E}^{j} / R^{j}}{N_{0}+\sum_{i \neq j} L^{i} \mathcal{E}^{i} / R^{i}}\right)-\frac{C^{j}}{R^{j}} .
$$

Consider now the (static) game $\mathbf{G}_{R}$ in which player $j$ chooses $R^{j} \in\left[s^{j}, S^{j}\right]$ so as to maximize $J^{j}\left(R^{j}\right)$. Let $Y^{j}:=1 / R^{j}$; it follows by the continuity and concavity of $h^{j}$ that $J^{j}$ is concave in $Y^{j}$ and continuous in $Y^{i}$ for $i \neq j$. Furthermore, $Y^{j}$ is chosen from a convex and compact set $\left[1 / S^{j}, 1 / s^{j}\right]$. It then follows by Theorem 1 in Rosen [8] that an equilibrium exists for $\mathbf{G}_{R}$. Noting that every equilibrium of $\mathbf{G}_{R}$ is an equilibrium point in our original game (by Lemma 3.1) concludes the proof.

We next show that the constant-power equilibrium described above is unique in some cases of interest.

Theorem 4.2: Assume that (i) $h^{j}$ is the identity function for every terminal $j$, or (ii) $h^{j}(x)=\log (1+x)$ for every terminal $j$. Then in either case there exists a unique equilibrium among those that have the structure described in Theorem 4.1 (i.e., the transmission power is constant in time).

Proof: As in the proof of Theorem 4.1, we consider the static game $\mathbf{G}_{R}$ where each terminal utility is given by (6). We then apply known uniqueness results for that $\mathbf{G}_{R}$. In particular, case (i) follows directly from [2] and case (ii) from [9], [10].

\section{EQUilibrium FOR THE Discrete-TIME MODEL}

Mixed Actions. In the discrete case, the action space is not convex since the times at which the battery can be changed can only take finitely possible values. Thus, a pure equilibrium will not exist in general in the discrete setting. We therefore consider a larger class of mixed (or of randomized) actions and do so in the context of the embedded model. However, we wish to avoid working with randomization over the infinite class of policies $\mathcal{A}^{j}$. Accordingly, we shall establish the existence of an equilibrium in which each terminal randomizes between a finite number of actions in $\mathcal{A}^{j}$.

Introduce the following class $\Delta_{a}^{j}$ of randomized actions. Any element of this class is a vector of the form $\left(\beta ;\left\{q_{k}\right\}, k=s^{j}, \ldots, S^{j}\right)$, where $\beta$ is a probability distribution over the finite set $\left\{s^{j}, \ldots, S^{j}\right\}$ and $q_{k} \in \mathcal{A}^{j}(k)$. A randomized action of terminal $j$ thus consists of a randomized choice $k$ of the time interval till the battery is changed next (the choice is done with probability $\beta(k)$ ) and of the vector $q_{k}$ of powers that the terminal uses during that interval. A policy $u^{j}$ for player $j$ is a sequence $\left(u_{1}^{j}, u_{2}^{j}, \ldots\right)$, where for each $m, u_{m}^{j}$ is a randomized action. We are particularly interested in the class of reduced stationary policies for each player $j$, denoted by $\Delta_{s}^{j}$; a policy belongs to this class if it uses at any time $t$ the same mixed action $v^{j}$, where $v^{j} \in \Delta_{a}^{j}$. 
We assume that each mobile $j$ does not to observe the actions of (nor the total interference from) other mobiles. Each mobile is assumed however to know the statistics of the total interference from other mobiles, so that the utility of the $j$ th mobile (4) becomes

$$
J^{j}(u)=\underline{\lim }_{T \rightarrow \infty} \frac{1}{T} E^{u}\left[\sum_{i=1}^{T} h^{j}\left(\operatorname{SINR}_{t}^{j}\right)-C^{j} \zeta_{T}^{i}\right],
$$

where $E^{u}$ is the expectation operator with respect to the vector of strategies $u=\left(u^{1}, \ldots, u^{N}\right)$.

Existence and Structure of the Equilibrium. We note that if mixed strategies are used by every mobile then each mobile is not faced anymore with fixed powers sent by other mobiles, so we cannot apply directly our previous results. However, by allowing mixed actions and consequently replacing utilities by their expectations, we shall be able to prove the existence of an equilibrium point, by applying similar steps as in the continuous-time case. To that end, it is required that the distribution of the interference for each terminal would be time invariant. We thus introduce the following assumption that will play the role of assumption Q2(j):

- Assumption Q3: Each player $j$ uses a policy under which $\left\{P_{t}^{j}\right\}$ is a stationary process. Moreover, the processes $\left\{P_{t}^{j}\right\}$ are independent from one player to another.

Lemma 5.1: Assume Q3. Then every terminal $j$ has an optimal response strategy for which the power process $\left\{P_{t}^{j}\right\}$ is stationary and independent of the processes $\left\{P_{t}^{i}\right\}$ for $i \neq j$.

Proof: Fix a strategy vector $u=\left(u^{j}, u^{-j}\right)$ satisfying $Q 3$, where $u^{j}$ is a strategy for player $j$ and $u^{-j}$ is the vector of strategies for the other players. Due to the independence of the processes $\left\{P_{t}^{i}\right\}$ corresponding to different players $i,(7)$ can be written similarly to (3), namely

$$
J^{j}(u)=\varliminf_{n \rightarrow \infty} E^{u} \frac{\sum_{m=1}^{n}\left[\sum_{t=\eta_{m}^{j}}^{\eta_{m+1}^{j}-1} h^{j}\left(\operatorname{SINR}_{t}^{j}\right)-C^{j}\right]}{\sum_{m=1}^{n} \tau_{m}^{j}}=\varliminf_{n \rightarrow \infty} E^{u^{j}} \frac{\sum_{m=1}^{n}\left(E^{u^{j}}\left[\mathcal{H}_{m}^{j}\left(u^{-j}\right) \mid \eta_{m}^{j}, \eta_{m+1}^{j}\right]-C^{j}\right)}{\sum_{m=1}^{n} \tau_{m}^{j}},
$$

where $\mathcal{H}_{m}^{j}\left(u^{-j}\right)=E^{u^{-j}}\left[\sum_{t=\eta_{m}^{j}}^{\eta_{m+1}^{j}-1} h^{j}\left(\operatorname{SINR}_{t}^{j}\right)\right]$. We note that the $J^{j}(u)$ depends on $\left\{P_{t}^{i}\right\}(i \neq j)$ only through their marginal distribution, and there is no dependence on joint distribution between the processes corresponding to players $i$ and $j$. Hence, for any best response of a player, the corresponding power process will indeed be independent of those of the other players (which is consistent with assumption Q3).

As in the proof of Lemma 3.1, the utility for terminal $j$ is maximized by maximizing $E^{u^{j}}\left[\mathcal{H}_{m}^{j}\left(u^{-j}\right) \mid \eta_{m}^{j}, \eta_{m+1}^{j}\right]$ for each $m$. We shall write the latter as $E^{u^{j}}\left[\mathcal{H}_{m}^{j}\left(u^{-j}\right) \mid \eta_{m}^{j}, \eta_{m+1}^{j}\right]=E^{u^{j}}\left[\sum_{t=\eta_{m}^{j}}^{\eta_{m+1}^{j}-1} \phi^{j}\left(P_{t}^{j}, u^{-j}\right) \mid \eta_{m}^{j}, \eta_{m+1}^{j}\right]$, where

$$
\phi^{j}\left(x, u^{-j}\right)=E^{u^{-j}}\left[h^{j}\left(\frac{L^{j} x}{N_{o}+\sum_{i \neq j} L^{i} P_{0}^{i}}\right)\right] .
$$

We note that $\phi^{j}$ is a concave function (since $h^{j}$ is). Proceeding exactly as in the proof of Lemma 3.1, we conclude that the constant power allocation $P_{t}^{j}=\frac{\mathcal{E}^{j}}{\tau_{m}^{j}}$ maximizes $E^{u^{j}}\left[\mathcal{H}_{m}^{j}\left(u^{-j}\right) \mid \eta_{m}^{j}, \eta_{m+1}^{j}\right]$ for each $m$.

It remains to derive the sequence $\left\{\eta_{m}^{j}\right\}$ and to show that this can be done in a way that the resulting process $\left\{P_{t}^{j}\right\}$ is stationary. A sufficient condition for optimality is that $\tau_{m}^{j} \in \mathcal{R}$, where $\mathcal{R}:=\operatorname{argmax}_{r \in\left[s^{j}, S^{j}\right]}\left\{\phi^{j}\left(\frac{\mathcal{E}^{j}}{r}, u^{-j}\right)-\frac{C^{j}}{r}\right\}$.

Let $\bar{\alpha}^{j}(r) \in \mathcal{A}^{j}(r)$ be the action in the embedded model that chooses a battery life time of $r$ and uses at each time unit the power $\mathcal{E}^{j} / r$. Let $u^{j}(q) \in \Delta_{a}^{j}$ be a randomized action that chooses at each time the battery is changed between one of the actions $\bar{\alpha}^{j}(r)$ with probability $q^{j}(r)$. Then if $u^{j}(q)$ is such that $q^{j}(r)>0$ if and only if $r \in \mathcal{R}$, it follows that $u^{j}(q)$ is a best response. Since the choices of $r$ are performed independently of each other, the whole process $\left\{P_{t}^{j}\right\}$ is stationary ergodic.

In the proof above, we have identified the best-response randomized action $u^{j}(q)$, which chooses at each time the battery is replaced a period $r$ (and a corresponding optimal power per slot of $\mathcal{E}^{j} / r$ ) with probability $q^{j}(r)$. We proceed to characterize the structure of $q^{j}(r)$. For a positive real number $\rho^{j}$, define $\rho^{j}$ to be the action that chooses 
a period of $r=\lfloor\rho\rfloor$ with probability $\rho-\lfloor\rho\rfloor$ and a period of $\lfloor\rho\rfloor+1$ otherwise. Note that $\rho \in\left[s^{j}, S^{j}\right]$. Denote by $\Lambda^{j}$ the set of actions $\rho^{j}$. We then have the following lemma.

Lemma 5.2: Assume Q3. Then every terminal $j$ has an optimal response strategy in $\Lambda^{j}$.

Proof: Follows directly from Lemma 5.1 and from the concavity in terms of $1 / r$ of

$$
J^{j}\left(u^{j}(q), u^{-j}\right)=E_{q^{j}(r)}\left[\phi^{j}\left(\frac{\mathcal{E}^{j}}{r}, u^{-j}\right)-\frac{C^{j}}{r}\right] .
$$

We next show that there always exists an equilibrium, where each terminal $j$ uses as stationary transmission strategy which alternates between at most two different periods $r$.

Theorem 5.3: There is an equilibrium point among the set $\Lambda^{1} \times \Lambda^{2} \cdots \times \Lambda^{N}$.

Proof: Assume that each terminal $j$ is limited to use a strategy in $\Lambda^{j}$. Note that $J^{j}\left(\rho^{j}, \rho^{-j}\right)$ is concave in $\rho^{j}$ (being a piecewise-linear concave function); it can be shown to be continuous in $\rho^{i}$ for $i \neq j$. Since $\rho^{j}$ is chosen from a convex and compact set $\left[s^{j}, S^{j}\right]$, there exists an equilibrium point in $\Lambda^{1} \times \Lambda^{2} \cdots \times \Lambda^{N}$ (by Theorem 1 in [8]). Using Lemma 5.2 this equilibrium remains so within the larger set of strategies $u=\left(u^{1}, \ldots, u^{N}\right)$. Indeed, since a best response always exists among $\Lambda^{j}$, no player can profit from a deviation to a strategy not in this class, and hence this is an equilibrium within the class of all strategies.

\section{Vi. Constrained Power Strategies}

In the previous sections we obtained optimal or equilibrium strategies that allocate the available battery energy uniformly in time. There may be cases, however, in which a constant power assignment is not possible. Some examples are:

- Assume that the maximum power $\bar{P}(\mathcal{E})$ that can be used decreases (in a continuous way) to zero as the amount of energy $\mathcal{E}$ remaining in the battery decreases to zero (this could be due to an increase in the internal resistance of the battery as it becomes old).

- Power levels may be discrete. This is the case, for example, in the UMTS standard for cellular telephony in Europe. If we want the battery to last time $T$ then the optimal power level obtained by dividing the total battery energy by $T$ need not coincide with one of the available levels.

It turns out that some weaker notions of regularity of power transmission policies could still be useful, and will allow us to obtain optimal or equilibrium strategies when constant powers cannot be implemented. To that end, we introduce the majorization order and the class of Schur concave functions. We provide below the basic definitions and theory that are required for our analysis.

Definition 6.1: (Majorization and Schur-Concave Function [11])

Consider two $n$-dimensional vectors $d(1), d(2)$. $d(2)$ majorizes $d(1)$, which we denote by $d(1) \prec d(2)$, if

$$
\sum_{i=1}^{k} d_{[i]}(1) \leq \sum_{i=1}^{k} d_{[i]}(2), \quad k=1, \ldots, n-1, \quad \text { and } \sum_{i=1}^{n} d_{[i]}(1)=\sum_{i=1}^{n} d_{[i]}(2),
$$

where $d_{[i]}(m)$ is a permutation of $d_{i}(m)$ satisfying $d_{[1]}(m) \geq d_{[2]}(m) \geq \ldots \geq d_{[n]}(m), m=1,2$.

A function $f: R^{n} \rightarrow R$ is Schur concave if $d(1) \prec d(2)$ implies $f(d(1)) \geq f(d(2))$.

Lemma 6.2: [11, Proposition C.1 on p. 64] Assume that a function $g: R^{n} \rightarrow R$ can be written as the sum $g(d)=\sum_{i=1}^{n} \psi\left(d_{i}\right)$ where $\psi$ is a concave function from $R$ to $R$. Then $g$ is Schur concave.

A General Framework for Adding Constraints. Assume that the set of actions available to each terminal is restricted through constraints, which may reflect technological limitations as exemplified above. Our goal is to identify best response policies for a player $j$ in the embedded decision model within $\Delta_{s}^{j}$. This new class need not contain policies that split the battery energy evenly along the battery's lifetime. We show below how Majorization allows to obtain alternative structure for best response and for equilibrium policies.

We focus throughout this section on the discrete-time setting. Assume that Q3 holds. A best response for mobile $j$ is obtained as in the proof of Lemma 5.1 in two steps: (i) For a given sequence $\left\{\eta_{m}^{j}\right\}$, compute an optimal power sequence to be used during the lifetime of the $m$ th battery; and (ii) Obtain an optimal sequence $\left\{\eta_{m}^{j}\right\}$. 
We mainly consider here the first step. Let $S^{j}(m)$ be the set of available power sequences of length $\tau_{m}^{j}$. Terminal $j$ then seeks for a sequence in $S^{j}(m)$ that maximizes the expression

$$
\sum_{t=\eta_{m}^{j}}^{\eta_{m+1}^{j}-1} \phi^{j}\left(P_{t}^{j}, u^{-j}\right)
$$

where $\phi^{j}\left(x, u^{-j}\right)$, given in Eq. (8), is concave. According to Lemma 6.2, the expression in (10) is Schur concave. Therefore any element of $S^{j}(m)$ that majorizes all others is optimal. We next show how the majorizing sequence can be efficiently obtained for the case where the transmission power in each slot is bounded as a function of the remaining energy.

An Example: Energy Dependent Constraints on the Maximum Power. Consider the case where the maximal transmission power that can be used in a given slot is a function of the remaining battery. As mentioned above, we are interested in optimizing performance for a given battery lifetime, say $s$. Accordingly, the relevant time under consideration is $t=1, \ldots, s$. Denote by $\bar{P}(e)$ the maximal power bound, where the energy is $e$. We assume that $\bar{P}(e)$ decreases in $e$. We further assume the following:

- Assumption Q4: For any player $j$, if a power assignment $Q_{t}^{j}$ is feasible, so is the assignment $R_{t}^{j}=Q_{[t]}^{j}$. In other words, if assignment $Q_{t}^{j}$ is feasible then so is the permutation of that assignment in a decreasing order.

Theorem 6.3: Assume Q4. Let $\mathcal{E}^{j}$ be the total energy of the battery. Consider sequences of length $s$, where $s$ is assumed to be such that all the energy of the battery can drain out during this period by some choice of a transmission policy. The optimal action for a sequence of length $s$ is given by the following procedure. Initiate $k:=s, \quad e_{k}:=\mathcal{E}^{j}$. While $k>0: P_{k}^{j}:=\bar{P}\left(e_{k} / k\right), \quad e_{k-1}:=e_{k}-P_{k}^{j}, \quad k:=k-1$.

Proof: Due to assumption Q4, it suffices to restrict attention to non-increasing sequences. We shall thus show that $P$ is majorized by any other non-increasing assignment, say $Q$. Noting that the minimal power per slot is at most $\mathcal{E}^{j} / s$, it follows by Assumption Q4 that

$$
P_{[s]}^{j} \geq Q_{[s]}^{j}
$$

Next we show that

$$
P_{s}^{j}+P_{s-1}^{j} \geq Q_{s}^{j}+Q_{s-1}^{j} \text {, implying that } P_{[s]}^{j}+P_{[s-1]}^{j} \geq Q_{[s]}^{j}+Q_{[s-1]}^{j} .
$$

Assume the contrary: $P_{s}^{j}+P_{s-1}^{j}<Q_{s}^{j}+Q_{s-1}^{j}$. Let $R$ be an assignment given by

$$
R_{k}^{j}=\left\{\begin{array}{lr}
Q_{k}^{j} & \text { for } k=1,2, \ldots, s-2 \\
Q_{s}^{j}+Q_{s-1}^{j}-P_{s}^{j} & \text { for } k=s-1 \\
P_{s}^{j} & \text { for } k=s .
\end{array}\right.
$$

Note that

$$
Q_{s-1}^{j} \geq R_{s-1}^{j}>P_{s-1}^{j},
$$

implying that $R_{s-1}^{j}=R_{[s-1]}^{j}$. Then since $P$ is lexicographic maximizer, and since $P_{s}^{j}=P_{[s]}^{j}=R_{s}^{j}=R_{[s]}^{j}$, it satisfies

$$
P_{s-1}^{j} \geq R_{s-1}^{j} .
$$

It follows from the above inequality that $R$ satisfies the power constraints at step $s-1$ : it clearly satisfies them for $t<s-1$ since $Q$ does, and it satisfies them at time $s$ since $P$ does. The inequalities (13) and (14) obviously contradict each other, thus (12) holds. Proceeding that way, we establish that $P$ is majorized by any other policy that satisfies the constraints.

The procedure suggested above relies on realizing that the transmission-bottleneck occurs at the last time slot. If it is impossible to assign equal powers to all slots then our recursion starts by assigning the largest value to the most constrained one. This assignment is also known as the max-min fair assignment.

Existence of Equilibrium. Equilibria policies are obtained by following similar arguments as in the previous section, except for one additional step. There is a possibility that we did not have before to obtain periodicity. If a policy $u^{j}$ is periodic then it need not be stationary. For example, if player $j$ uses only one value for all $\tau_{m}^{j}$, a periodic sequence $\left\{P_{t}^{j}\right\}$ can be generated. This can be avoided by shifting the policy by a phase uniformly distributed over $\left\{0, . ., \tau_{m}^{\}}-1\right\}$. 


\section{AdDitional USER UTILITIES}

In this section we consider other user utilities, besides the additive utility (2). Our main focus here is on the Bit-per-Joule utility, which has been widely used as a performance criterion in shared wireless networks (see [12] and references therein). We next consider this utility under the continuous time model, given by

$$
\widehat{J}^{j}(u):=\underline{\lim }_{T \rightarrow \infty} \frac{1}{\mathcal{E}^{j} \zeta_{T}^{j}} \cdot \int_{t=0}^{T} h^{j}\left(\operatorname{SINR}_{t}^{j}\right) \mathrm{d} t
$$

As in previous sections, assume that all users but the $j$ th one transmit with constant powers. Further assume that user $j$ uses a periodic strategy (there is obviously no advantage in modifying the transmission strategy for different batteries). Then (15) is equivalent to

$$
\widehat{J}^{j}\left(R^{j}\right):=\varliminf_{n \rightarrow \infty} \frac{n \int_{t^{\prime}=0}^{R^{j}} h^{j}\left(S I N R_{t^{\prime}}^{j}\right) \mathrm{d} t^{\prime}}{n \mathcal{E}^{j}},
$$

where $R^{j}$ is the period used by user $i$ and $t^{\prime} \in\left[0, R^{j}\right]$ is the time index within a period. We can now use the same argument as in Lemma 3.1, to conclude that there exists a best-response of user $j$ which consists of constant-power transmission. Consequently, after some simple algebra, (16) can be written as $\widehat{J}^{j}\left(R^{j}\right)=R^{j} \tilde{h}^{j}\left(1 / R^{j}\right)$, where $\tilde{h}^{j}$ is a concave function, derived from the original $h^{j}$, which incorporates the use of constant powers by all users. Differentiation of the last equation with respect to the period $R^{j}$ yields $\frac{\partial \widehat{J}^{j}\left(R^{j}\right)}{\partial R^{j}}=\tilde{h}^{j}\left(1 / R^{j}\right)-\left(1 / R^{j}\right) \tilde{h}^{j^{\prime}}\left(1 / R^{j}\right)$. Replacing $Y^{j}=\left(1 / R^{j}\right)$ and noting that $\tilde{h}^{j}(0)=0$, we use the gradient inequality [13] for the above derivative to conclude that $\frac{\partial \widehat{J}^{j}\left(R^{j}\right)}{\partial R^{j}} \geq 0$. This inequality is strict if $\tilde{h}^{j}$ is strictly concave. Our analysis therefore suggests that when mobiles adopt (15) as their utility, there is an equilibrium point where all users transmit at constant, low powers, which correspond to the upper bound on their periods, $P^{j}=\frac{\mathcal{E}}{S^{j}}$. If there is no effective upper bound on the period, users are better off transmitting at a power of zero. The above discussion indicates that the use of the bit per joule as a performance criterion, may lead to inefficiency in network utilization under a distributed framework. We note that if a Sigmoid function of the SINR is used instead of a concave function (see [12]), other equilibria are possible. We are currently investigating this extension.

One natural extension to the user model and to the utilities involved is to consider utilities which are a function not only of the SINR but also of the remaining battery. For example, a mobile user may consider its data rate more valuable when the battery is about to drain out. This extension incorporates novel theoretic challenges and is an interesting subject for future research.

\section{ASYMMETRIC STRATEGIES}

The equilibria we have identified so far have consisted of regular power transmission strategies. In the continuous time model they were in fact constant in time. We have also shown that the equilibrium among those strategies can be unique. We next show that the equilibrium is not unique in general; other equilibria that are not constant nor regular may exist.

To illustrate this, assume that we have $J$ terminals each with $\mathcal{E}_{j}$ energy units available for transmission during a period of duration $s^{j}=S^{j}=s$. Define the following policies.

- TDMA policy Divide $s$ into $J$ disjoint time slots with durations $T^{1}, T^{2}, \ldots, T^{J}$ with $\sum_{j} T^{j}=s$. Let player $j$ transmit during slot $j$ all its energy $\mathcal{E}^{j}$. (slot durations need not be equal; the transmission power during a slot need not be identical to all users).

- Randomized $\epsilon$-impulse policies Each terminal $j$ chooses independently of other terminals a random time $t^{j}$. For some small $\epsilon$, it transmits all its energy during the time interval $\left[t^{j}, t^{j}+\epsilon\right]$.

Consider the special case where $h^{j}$ is the identity function (which means that terminals seek to maximize their SINRs). It can be easily shown that the TDMA policy is both an equilibrium policy as well as an overall optimal one. We note however that TDMA requires a synchronization or a coordination mechanism in order to avoid overlaps in the choices of slots. The equilibrium in presence of such a coordination is the well known correlated equilibrium. In the absence of a coordination mechanism, one can use the Randomized $\epsilon$-impulse policy. As $\epsilon$ tends to the limit zero, the policy converges to an equilibrium one.

Consider also the TDMA policy for the case where $h^{j}(x)=\log (1+x)$ for every terminal $j$. Assume that the terminals are symmetric with respect to both their energy $\left(\mathcal{E}_{j}=\mathcal{E}\right)$, channel gains and transmission periods. For 
simplicity, let $L^{j}=1, N_{0}=1$ and $T^{j}=1$ for every user $j$. We next show that TDMA is an equilibrium policy in this case as well. To that end, we ignore the recharge cost, as it can not be optimized due to the fixed period. The utility per-period under TDMA is $\log (1+\mathcal{E})$. If TDMA is not an equilibrium, then some user can shift power of $0<\delta \leq \mathcal{E}$ to some other slot and improve its utility. This action will result in a utility of $\log (1+\mathcal{E}-\delta)+\log \left(1+\frac{\delta}{1+\mathcal{E}}\right)$. However, noting that

$$
\log (1+\mathcal{E}-\delta)+\log \left(1+\frac{\delta}{1+\mathcal{E}}\right)=\log \left(\frac{(1+\mathcal{E}+\delta)(1+\mathcal{E}-\delta)}{1+\mathcal{E}}\right)=\log \left(1+\mathcal{E}-\frac{\delta^{2}}{1+\mathcal{E}}\right)<\log (1+\mathcal{E}),
$$

we conclude that the utility can not be improved, hence the TDMA policy is an equilibrium one.

\section{CONCLUSION}

We have investigated the interaction between self-interested wireless users, each wishing to maximize its throughput utility with minimal battery-recharge costs. We studied both continuous and discrete time models. We obtained interesting structure of equilibrium policies, and in particular, in case where the transmission power may depend on the remaining battery energy.

We have shown that time-sharing equilibria may exist for certain utility functions. An interesting research direction is to examine whether additional asymmetric equilibria do exist. One may consider other reception models beyond the CDMA-based network studied here. In particular, capture models, which sometimes better represent Wireless LAN systems are of great interest. In these models, the base station can properly receive the signal of a single station at a time. Consequently, the use of constant powers by all terminals, is inherently not an equilibrium point. Hence, new solution approaches would have to be obtained.

\section{REFERENCES}

[1] C. Saraydar, N. N.B. Mandayam, and D. Goodman, "Efficient power control via pricing in wireless data networks," IEEE Transactions on Communications, vol. 50, no. 2, pp. 291-303, 2002.

[2] T. Alpcan, T. Basar, R. Srikant, and E. Altman, "CDMA uplink power control as a noncooperative game," Wireless Networks, vol. 8, no. 6, pp. 659-670, 2002.

[3] G. Scutari, S. Barbarossa, and D. P. Palomar, "Potential games: A framework for vector power control problems with coupled constraints," in Proc. IEEE International Conference on Acoustics, Speech, and Signal Processing (ICASSP), 2006.

[4] C. W. Sung and W. S. Wong, "A noncooperative power control game for multirate CDMA data networks," IEEE Transactions on Wireless Communications, vol. 2, no. 1, pp. 186 - 194, Jan 2003.

[5] L. Lai and H. El-Gamal, "The water-filling game in fading multiple access channel," IEEE Transactions on Information Theory, 2006.

[6] E. Altman, K. Avrachenkov, G. Miller, and B. Prabhu, "Discrete power control: Cooperative and non-cooperative optimization," in INFOCOM, 2007.

[7] D. Fudenberg and J. Tirole, Game Theory. MIT Press, 1991.

[8] J. B. Rosen, "Existence and uniqueness of equilibrium points for concave n-person games," Econometrica, vol. 33(3), pp. 520-534, 1965.

[9] Z. Q. Luo and J. S. Pang, "Analysis of iterative waterfilling algorithm for multiuser power control in digital subscriber lines," Eurasip J. on Applied Signal Processing, vol. Article ID 24012, pp. 1-10, 2006.

[10] L. Pavel, "A noncooperative game approach to OSNR optimization in optical networks," IEEE Trans on Automatic Control, vol. 52, no. 5, pp. 848-852, 2006.

[11] A. W. Marshall and I. Olkin, Inequalities: Theory of Majorization and its Applications. Academic Press, 1979, vol. 143 of Mathematics in Science and Engineering.

[12] F. Meshkati, H. V. Poor, and S. C. Schwartz, "Energy-efficient resource allocation in wireless networks," IEEE Signal Processing Magazine, vol. 24, no. 3, 2007.

[13] S. Boyd and L. Vandenberghe, Convex Optimization. Cambridge University Press, 2003. 